\title{
OGLE-TR-3: A possible new transiting planet ${ }^{\star}$
}

\author{
S. Dreizler ${ }^{1}$, P. H. Hauschildt ${ }^{2}$, W. Kley ${ }^{3}$, T. Rauch ${ }^{1,4}$, S. L. Schuh ${ }^{1}$, K. Werner ${ }^{1}$, and B. Wolff ${ }^{5}$ \\ 1 Institut für Astronomie und Astrophysik, Abt. Astronomie, Sand 1, 72076 Tübingen, Germany \\ 2 Hamburger Sternwarte, Gojenbergsweg 112, 21029 Hamburg, Germany \\ 3 Institut für Astronomie und Astrophysik, Abt. Computational Physics, Auf der Morgenstelle 10, 72076 Tübingen, Germany \\ ${ }^{4}$ Dr.-Remeis-Sternwarte, Sternwartstraße 7, 96049 Bamberg, Germany \\ 5 European Southern Observatory, Karl-Schwarzschild-Straße 2, 85748 Garching, Germany
}

Received 20 December 2002 / Accepted 19 February 2003

\begin{abstract}
Recently, 59 low-luminosity object transits were reported from the Optical Gravitational Lensing Experiment (OGLE). Our follow-up low-resolution spectroscopy of 16 candidates provided two objects, OGLE-TR-3 and OGLE-TR-10, which have companions with radii compatible with those of gas-giant planets. Further high-resolution spectroscopy revealed a very low velocity variation $\left(<500 \mathrm{~m} \mathrm{~s}^{-1}\right)$ of the host star OGLE-TR-3 which may be caused by its unseen companion. An analysis of the radial velocity and light curve results in $M<2.5 M_{\text {Jup }}, R<1.6 R_{\mathrm{Jup}}$, and an orbital separation of about $5 R_{\odot}$, which makes it the planet with the shortest period known. This allows to identify the low-luminosity companion of OGLE-TR-3 as a possible new gas-giant planet. If confirmed, this makes OGLE-TR-3 together with OGLE-TR-56 the first extrasolar planets detected via their transit light curves.
\end{abstract}

Key words. binaries: eclipsing - stars: individual: OGLE-TR-3 - stars: low-mass, brown dwarfs - stars: planetary systems

\section{Introduction}

The detection of planets outside the solar system was a longstanding goal of astronomy. After the first successful detections (Wolszczan \& Frail 1992; Mayor \& Queloz 1995), the search with various methods (see Schneider 2001 for an overview) was largely intensified. Out of the 104 presently known planets, 100 have been detected with Doppler-velocity measurements of the planets' host stars. All these planets were found around solar-like stars. The other four are planets around pulsars and were found by periodic pulse-modulation measurements (see The Extrasolar Planets Encyclopaedia http://www.obspm.fr/encycl/catalog.html for an upto-date overview).

Until very recently, no planet had yet been found by photometric monitoring. HD 209458 was the only system which has an orbital inclination that allows the measurement of the eclipse of the host star by the planet (Charbonneau et al. 2000; Henry et al. 2000). This planetary companion was, however, known before from Doppler measurements (Mazeh et al. 2000). The current paucity of extrasolar planets found via the transit method is deplorable for several reasons. The transiting systems provide more reliable parameters. With the known inclination, the radial velocity variation provides a precise mass

Send offprint requests to: S. Dreizler

e-mail: dreizler@astro.uni-tuebingen.de

* Based on observations collected at the European Southern Observatory, Paranal, Chile (ESO Programme 269.C-5034). determination and not only a lower mass limit. Together with the radius, the density of the planet can additionally be derived. The transit also opens, at least in principle, the possibility to explore the planet's atmosphere during the eclipse (Charbonneau et al. 2002). Furthermore, transits are the most promising way to detect earth-like planets around solar-like stars and it is therefore the method of choice for several ground and space based projects.

Recently, 59 transiting planet candidates were announced by the Optical Gravitational Lensing Experiment (OGLE) consortium (Udalski et al. 2002a,b). These candidates were extracted from a sample of about 5 million stars observed during a 32-day photometric monitoring. In a sub-sample of 52000 stars with a photometric accuracy better than $1.5 \%$, these 59 candidates exhibit light curves indicating the presence of a transiting low-luminosity companion. From this sample we obtained low-resolution spectra for 16 objects (Dreizler et al. 2002) in order to derive spectroscopic radii of the primary stars and together with the eclipse light curve also the companion radii. While 14 low-luminosity companions could be identified as M stars, two objects, namely OGLE-TR-3 and OGLE-TR-10, have companions with radii compatible with those of gas-giant planets. OGLE-TR-10 has been further investigated by Hatzes et al. (ESO Programme 268.C-5772). We will present in this paper our VLT follow-up observations for a dynamical mass determination of the companion of OGLE-TR-3.

Very recently, Konacki et al. (2003) claimed the verification of the planetary nature of OGLE-TR-56. They also 
investigated OGLE-TR-3, OGLE-TR-10, OGLE-TR-33, and OGLE-TR-58. While the interpretation as planetary host star for none of these objects is conclusive from their work yet, they regard OGLE-TR-3 as the result of blending and grazing eclipse in contrast to our interpretation.

In the following, we describe our observations and data reduction (Sect. 2). The analysis is presented in Sect. 3 and the results are discussed in Sect. 4.

\section{Observations and data reduction}

The aim of the observations is a detection of the reflex motion of OGLE-TR-3 due to the low-luminosity companion causing the observed eclipse light curve (Udalski et al. 2002a). The short orbital period $(P=1.1899$ days $)$ in combination with a spectroscopic mass of $1.1 M_{\odot}$ (Dreizler et al. 2002), a lowmass star $\left(M>0.08 M_{\odot}\right)$ would produce a radial velocity variation of the order of $10 \mathrm{~km} \mathrm{~s}^{-1}$. A discrimination between a stellar and sub-stellar object therefore needs only moderate instrument requirements. We observed in Service Mode with UVES mounted at the VLT Kueyen. We used the red arm of the spectrograph with a central wavelength of $5800 \AA$, a slit width of 0.7 , and the CCD detectors without binning. Image slicer \#1 was inserted in order to obtain the full spectral resolution of 60000 independently of the seeing.

We were granted one night of Directors Discretionary Time which we spread in ten observing blocks of each $3 \times 20$ min exposures to minimize the velocity smearing due to the earth's motion. We asked for five consecutive nights with each two observing blocks around the meridian passage of OGLE-TR-3 in order to sample the orbital period equidistantly. Due to bad weather and other targets of opportunity this schedule could not be kept and observations were finally spread out over about one month (for details, see Table 1).

The spectra were extracted using procedures of the UVES context of the ESO-MIDAS software package. The routines provide correction for bias, flat-field, and background level. Wavelength calibration was performed with ThAr spectra obtained immediately after each observing block. According to the instrument handbook this provides a precision to about $100 \mathrm{~m} \mathrm{~s}^{-1}$.

From the exposure time calculator we expected a maximum $S / N$ of about 15 in a $20 \mathrm{~min}$ exposure. The achieved $S / N$ was significantly lower (around 10). We therefore had to co-add the three individual frames of one observing block to each one single exposure. As an example, we show a small section of the co-added spectrum and of all spectra from ten observing blocks (Fig. 1) overlayed with a synthetic spectrum from our spectral analysis (see below).

\section{Analysis}

\subsection{Spectral analysis}

An inspection of the VLT-UVES spectra of OGLE-TR-3 reveals three spectral components. The earth's atmosphere component, consisting of the night sky emission and telluric lines,

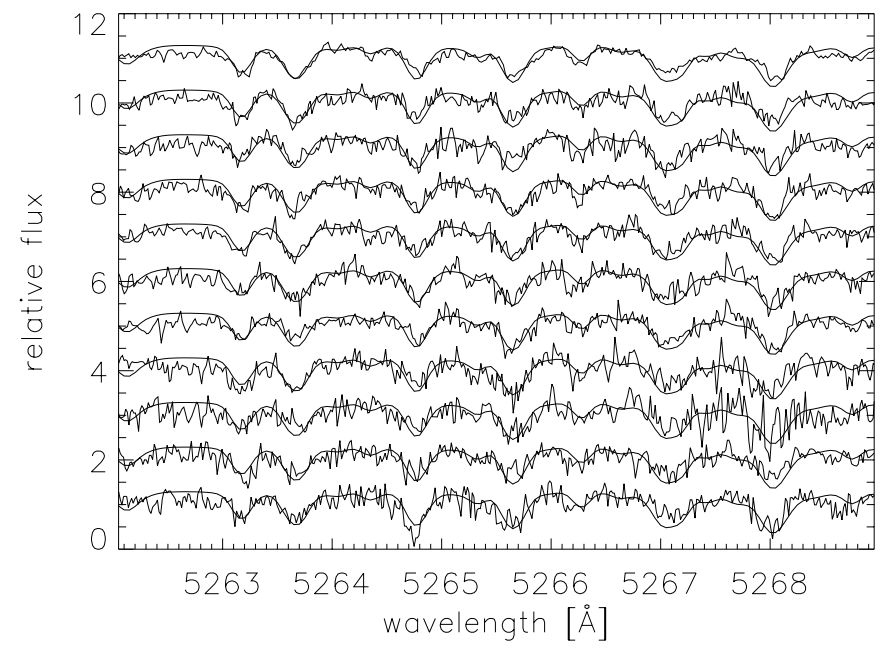

Fig. 1. From top to bottom: a small section of the co-added OGLE-TR-3 spectrum and the individual spectra of the ten observing blocks ( $1 \mathrm{~h}$ observing time each) ordered by JD as listed in Table 1 .

are easy to separate since they do not follow the radial velocity shifts due to the earth's orbital motion. Besides the stellar absorption lines, there is a third component, mainly visible in the NaD doublet at about $5890 \AA$, which has a constant velocity offset of $20 \mathrm{~km} \mathrm{~s}^{-1}$. The second absorption component could be caused by a stellar companion of OGLE-TR-3 or by interstellar absorption. If this were due to a binary companion we could use the difference in radial velocity and interpret it as orbital velocity. With the parameters of OGLE-TR-3 this would result in an orbital period of about 4 years. This component can therefore not be responsible for the observed 1.1899 day eclipse period. If identified as binary companion we would expect to see other spectral features at that velocity shift, if it is also a late $F$ to early G star as OGLE-TR-3. This is, however, not the case. The possible stellar companion could alternatively be of later spectral type, down to late K. It would then be about a factor of two fainter. Since $\mathrm{NaD}$ is among the strongest spectral features for those spectral types, this would explain why we cannot detect other lines. We should, however, detect the $\mathrm{MgH}$ band around $5100 \AA$, which is not the case.

As demonstrated in Fig. 2, we can fit the NaD line nicely with an interstellar absorption caused by a column density of $5 \times 10^{15} \mathrm{~cm}^{-2}$ neutral $\mathrm{Na}$ atoms. This is a very plausible value since OGLE-TR-3 lies about $3 \mathrm{kpc}$ in the direction of the galactic bulge.

We also investigated the possibility of blending, first due to a second, equally luminous star at the velocity of OGLE-TR-3. This hypothetical third object would then be a late $\mathrm{F}$ to late $\mathrm{G}$ star, since an earlier spectral type would dominate the spectrum and a later one would hardly contribute to the total brightness. The eclipse could then result from a late K or early M star orbiting one of the $\mathrm{G}$ stars, the shallow eclipse would just be a fake due to the blend $(\mathrm{G}+[\mathrm{G}+\mathrm{M}]$ scenario in the following), as suggested by Konacki et al. (2003). We therefore carefully inspected our spectra in order to detect asymmetries in the line profiles. As demonstrated in Fig. 1 with the overlayed synthetic spectrum, all individual spectra appear symmetric and the 


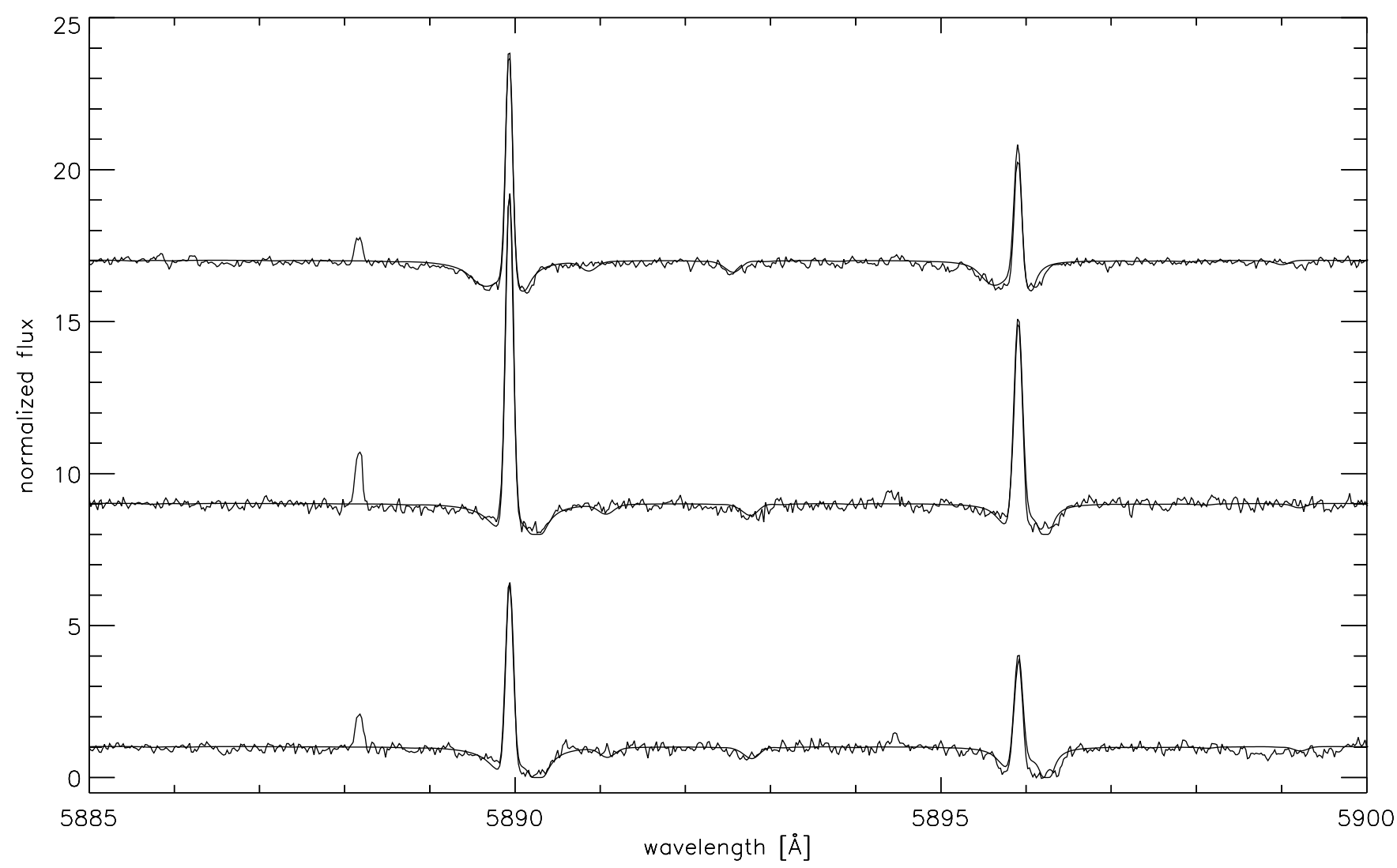

Fig. 2. Three normalized VLT-UVES spectra of OGLE-TR-3, obtained at JD-2452400 $=62.61590,86.57832$, and 89.56877 (from bottom to top), around the $\mathrm{NaD}$ doublet. The fit consists of three components: A synthetic spectrum shifted by the measured radial velocity (blue wing), a Gaussian emission component from the earth's atmosphere, and an interstellar absorption of a column density of $5 \times 10^{15} \mathrm{~cm}^{-2}$ neutral Na atoms for the red wing. The velocity of the interstellar component has a fixed offset of $+20 \mathrm{~km} \mathrm{~s}^{-1}$ relative to the stellar component.

Table 1. Observing dates, orbital phase of OGLE-TR-3, measured radial velocity relative to a co-added spectrum, earth's orbital and earth's rotational velocity components towards OGLE-TR-3, radial velocity of OGLE-TR-3, and standard deviation of the measurements. The radial velocity column provides the measured velocity corrected for earth's motions minus the median of the 10 values.

\begin{tabular}{rl|rrrrr}
\hline \hline JD-2452400 phase & \multicolumn{5}{|c}{ radial velocity $\left[\mathrm{km} \mathrm{s}^{-1}\right]$} \\
& & spec. & orbit & rot. & \multicolumn{1}{c}{ TR-3 } & $\sigma$ \\
\hline 62.61590 & 0.1754 & 0.06 & -8.32 & 0.11 & 0.151 & 0.12 \\
62.66215 & 0.2143 & 0.15 & -8.34 & 0.01 & 0.116 & 0.17 \\
63.63365 & 0.0307 & 0.51 & -8.80 & 0.07 & 0.072 & 0.10 \\
63.67882 & 0.0687 & 0.56 & -8.82 & -0.03 & -0.003 & 0.16 \\
66.61967 & 0.5400 & 2.11 & -10.20 & 0.08 & 0.290 & 0.25 \\
66.66758 & 0.5804 & 1.95 & -10.22 & -0.02 & -0.004 & 0.13 \\
86.53247 & 0.2741 & 10.11 & -18.65 & 0.15 & -0.094 & 0.58 \\
86.57832 & 0.3127 & 10.32 & -18.67 & 0.05 & 0.000 & 0.64 \\
89.52445 & 0.7885 & 11.14 & -19.77 & 0.15 & -0.192 & 0.44 \\
89.56877 & 0.8257 & 11.13 & -19.79 & 0.06 & -0.306 & 0.55 \\
\hline
\end{tabular}

co-added spectrum does not show any signs of additional broadening. The effect, if present, is expected to be directly visible since an $M$ star companion to a $G$ star in the short orbital period of OGLE-TR-3 would result in a radial velocity variation of the order of $10 \mathrm{~km} \mathrm{~s}^{-1}$ corresponding to a one pixel shift on the detector. This would also be visible in the co-added spectrum as additional broadening of the same order of magnitude. This is not the case (see below). For a more quantitative investigation, we fitted 17 strong and unblended spectral lines with Gauss profiles and calculated the difference between the observations and the Gauss fit for the red and blue wings separately. The total difference is shown in Fig. 3. Any asymmetry would result in systematic offsets between the red and blue line wings. Additionally we cannot find a trend with the orbital period. Our current data set provides no hint for blending and we therefore regard the scenario proposed by Konacki et al. (2003) as unlikely in case of OGLE-TR-3.

The second blending scenario is a close $\mathrm{M}+\mathrm{M}$ star binary orbiting at $2 \times 1.1899$ days blended by a $G$ star with a large enough distance towards the $\mathrm{M}$ star binary so that it has an undetectable orbital velocity $(\mathrm{G}+[\mathrm{M}+\mathrm{M}]$ scenario in the following). We inspected our UVES spectra in order to detect spectral features from a possible $\mathrm{M}$ star. There is no evidence for it but since the contribution of two M stars is only of the order of $3 \%$ in the red, we cannot rule out this completely. The light curve from such a system is discussed below (Sect. 3.3).

The third blending scenario is a line of sight blending: a G0 star is contributing most of the light while an eclipsing binary system is situated at a much larger distance $(\mathrm{G}+[X+Y]$ in the following). Its contribution to the spectrum must be (very 


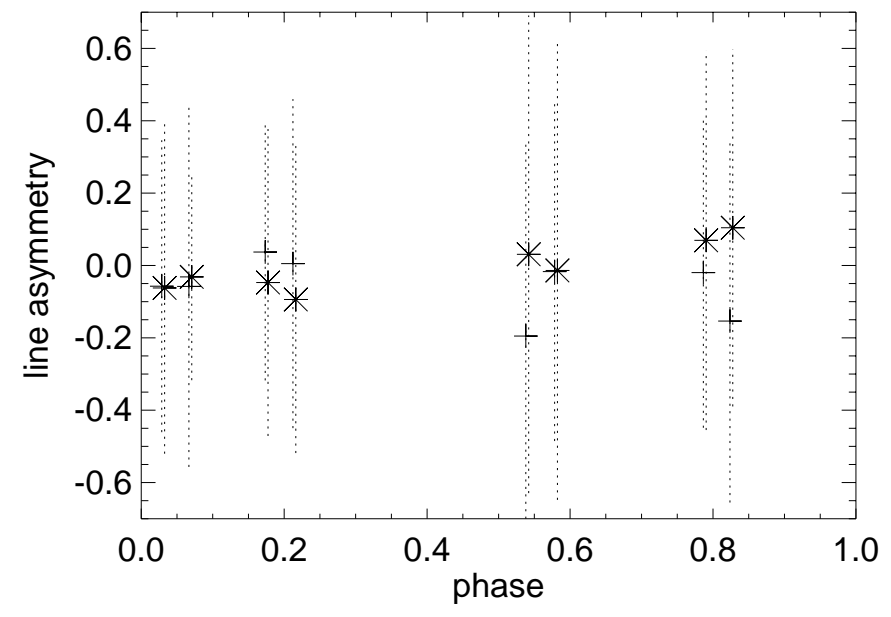

Fig. 3. Co-added differences between red "+" and blue "*" wings of observed spectral lines and Gauss profiles with $1 \sigma$ error bars. The spectra from $\mathrm{JD}=86.53247$ and 86.57832 are omitted due to very large uncertainties. No indication of line asymmetry is present in our data set.

conservatively) less than about $30 \%$ because we would detect it otherwise, as discussed above. Its influence on the light curve is also discussed below (Sect. 3.3).

We fit the co-added spectrum of OGLE-TR-3 by comparing it to a PHOENIX (version 13.00) spherical model atmosphere grid and synthetic spectra calculated at the resolution of the observed spectrum (Figs. 1 and 4). The model atmosphere grid covers a wide range of effective temperatures, gravities, and abundances. It includes the latest updates with respect to the NextGen (Hauschildt et al. 1999) and Dusty/Cond (Allard et al. 2001) grids. For simplicity, we have used LTE models, since NLTE effects in the relevant parameter range can be expected to be small and were thus neglected for the initial fits. The updates include improvements in the equation of state data used and better molecular line lists, however, the latter are not important for OGLE-TR-3.

The synthetic spectra grid covers a range of $6000 \mathrm{~K} \leq$ $T_{\text {eff }} \leq 6200 \mathrm{~K}$ and $4.0 \leq \log g \leq 5.0$ for solar abundances and $[\mathrm{M} / \mathrm{H}]=-0.5$, in agreement with our earlier spectral classification as F9/G0 V star (Dreizler et al. 2002). Synthetic spectra were computed with $0.01 \AA$ steps and rotationally broadened for $v_{\text {rot }} \sin i=2 \mathrm{~km} \mathrm{~s}^{-1}$. Again, this is a clear indication that no third object as in the $\mathrm{G}+[\mathrm{G}+\mathrm{M}]$ scenario is present, as discussed above. In addition, the spectral resolution was downgraded to $0.02 \AA$ before comparison with the data. The observed spectra are not flux calibrated, so the synthetic spectra were normalized to the continuum. With this procedure, we find that the best fit parameters for OGLE-TR-3 are $T_{\text {eff }}=$ $6100 \pm 200 \mathrm{~K}, \log g=4.5 \pm 0.5$ and abundances between solar and $[\mathrm{M} / \mathrm{H}]=-0.5$. We did not attempt to improve the fit by changing the abundance pattern, but inspection of the comparisons shows that this could lead to improved fits. Models outside the parameter range do not lead to improved fits, and were not considered further. In Fig. 5 we compare these parameters with stellar evolution models (Schaerer et al. 1993) and derive the stellar mass and radius. The spectral analysis corroborated the stellar parameters of the primary derived earlier $\left(R=1.05 R_{\odot}, M=1.1 M_{\odot}\right.$ Dreizler et al. 2002). However, our fit favors slightly lower parameters, $R=1.0_{-0.2}^{+0.9} R_{\odot}$ and $M=1.0_{-0.1}^{+0.3} M_{\odot}$. We adopt $R=1.0 R_{\odot}$ and $M=1.0 M_{\odot}$ in the following radial velocity and light curve analysis.

\subsection{Radial velocity variation}

For the determination of the radial velocity variation of OGLE-TR-3 as reflex motion due to the unseen companion, we used the first spectrum as reference and determined the crosscorrelation in velocity space relative to the other nine. We identified twelve $50 \AA$ intervals within the available spectral range (4800-6800 §) with little contamination from telluric lines. Additionally, we had to avoid the spectral region between 5750 and $5850 \AA$ where the spectrum falls onto the gap between the two CCDs. The cross-correlation function is approximated by a Gauss profile to determine the velocity shift at sub-pixel precision (see Fig. 6). The median of the twelve segments is then taken as the velocity shift of the spectrum. The standard deviation is used as quality attribute of the measurement and directly reflects the $S / N$ of the spectra. Afterwards we co-added all spectra in velocity space and repeated the procedure, now with the co-added spectrum as reference. Reassuringly, neither the cross-correlation with the co-added spectrum nor with a model spectrum does change the results significantly.

Using the period ( $P=1.1899$ days) and epoch of the mid eclipse (JD = 2452060.22765) from Udalski et al. (2002a), we calculated the phasing of each mid of exposure ${ }^{1}$. For each observation we also calculated the velocity components of the earth towards OGLE-TR-3 using the BARYVEL routine available in the Interactive Data Language (IDL) astrolib from Goddard Space Flight Center. It should be noted, that this routine provides the velocity in a right-handed coordinate system with the $+X$ axis toward the vernal equinox, and $+Z$ axis toward the celestial pole. A positive velocity component towards an object therefore produces a blue shift whereas the spectroscopic definition for the sign of the velocity is the opposite. We also calculated the velocity components due to the earth's rotation, which is, however, of minor importance. In Table 1 we summarize our results for each exposure.

An independent period determination is impossible from our few scattered measurements. Hence we mapped the radial velocity curve to the orbital phase (Fig. 7) using the period and zero point from Udalski et al. (2002a). These data were fitted with a sine curve with an offset, the velocity amplitude, and a phase shift as free parameters. We can use the fitted phase as an indication that the period is present in our data. As expected, the derived radial velocity is indeed zero at mid eclipse.

First, we used an inversion based $\chi^{2}$-minimization routine. Figure 7 shows the measured radial velocities with error bars of $1 \sigma$ overlayed with the best fit (solid). We also show the velocity curve for $3 \sigma$ variation (dashed) in the amplitude (see also Table 2). The resulting amplitude of about $100 \mathrm{~m} \mathrm{~s}^{-1}$ is smaller

1 The slightly different values given at the OGLE-web site http://sirius.astrouw.edu.pl/ ogle result in the same conclusions. 

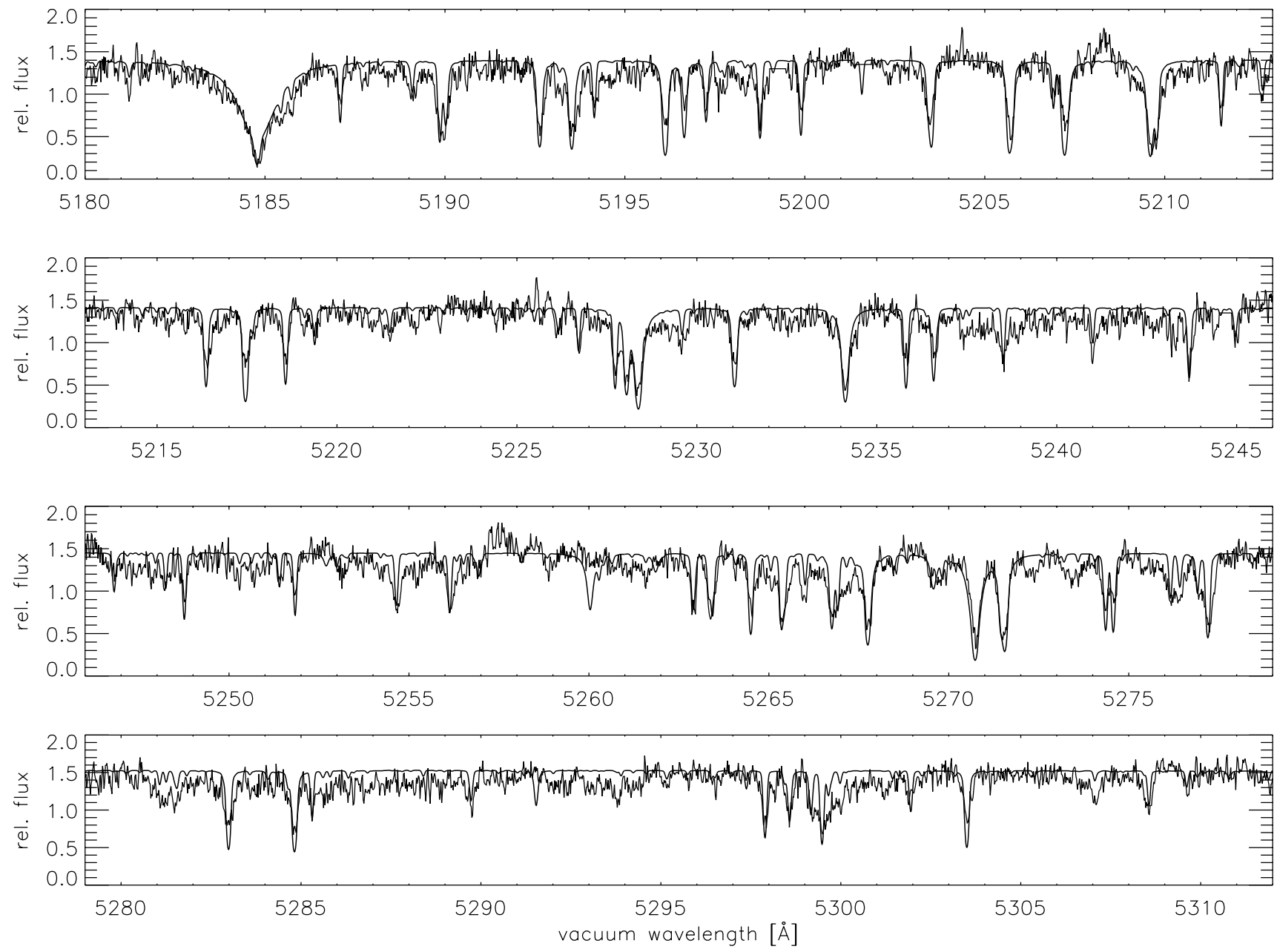

Fig. 4. Four $50 \AA$ sections of the co-added spectrum of OGLE-TR-3 compared to a theoretical model: $T_{\text {eff }}=6100 \mathrm{~K}, \log g=4.5$ (cgs), and sub-solar abundances $([\mathrm{M} / \mathrm{H}]=-0.5)$.

than the median $1 \sigma$ error, we therefore investigate the significance of the result in more detail.

We used night-sky emission lines which do not show velocity variation due to the earth's motion. The resulting "velocity" variation (Fig. 8) reveals a fit with vanishing amplitude, as expected. It also demonstrates that we recover the velocity precision of UVES $\left(\approx 100 \mathrm{~m} \mathrm{~s}^{-1}\right)$. This test is an indication that the derived velocity amplitude is indeed due to intrinsic variations rather than to measurement errors.

Additionally, we utilized the genetic algorithm pikaia (Charbonneau 1995) to confirm the three fit parameters. The genetic algorithm starts from randomly distributed parameter sets and increases the quality of the fit according to selection criteria adopted from the biological process of evolution. It therefore provides the global $\chi^{2}$-minimum much more reliably than classical $\chi^{2}$-minimization. In order to obtain a measurement for the reliability of the result, we ran pikaia with 1000 different initializations of the starting population. The deviation of the final fit parameters is very small (Table 2) and they are consistent with the one of the classical $\chi^{2}$-minimization.

As a third test, we synthesized 1000 white noise curves with the variance of that from the radial velocity curve and with identical timing and fitting weights. These data sets were fitted with pikaia. In Fig. 9 we compare the results of the pikaia fit of the radial velocity curve with those from the simulations. While the parameters of the radial velocity variations are recovered with a very small scatter, the noise simulations produce fit results over a large parameter space. While the fitted amplitudes and offsets are typically of the order of the mean standard deviation of our measurements $\left(\approx 200 \mathrm{~m} \mathrm{~s}^{-1}\right)$, the phase fits are equally distributed over the interval $[0,1]$. As measurement for the reliability of the fit, we provide the widths of the parameter interval for the radial velocity fits divided by the width of the simulation fits.

Concluding, we can fit the observed radial velocity curve with an amplitude of about $120 \mathrm{~m} \mathrm{~s}^{-1}$. We provided several indications that this fit indeed represents a true reflex motion of the late $\mathrm{F} /$ early $\mathrm{G}$ type planetary host star due to the unseen companion. This contradicts the $\mathrm{G}+[\mathrm{M}+\mathrm{M}]$ scenario, where the $G$ star would have a very small radial velocity variation at a much larger orbital period.

\subsection{The light curve revisited}

For a consistency check, we fitted the light curve of OGLE-TR-3 published by Udalski et al. (2002a) with our 

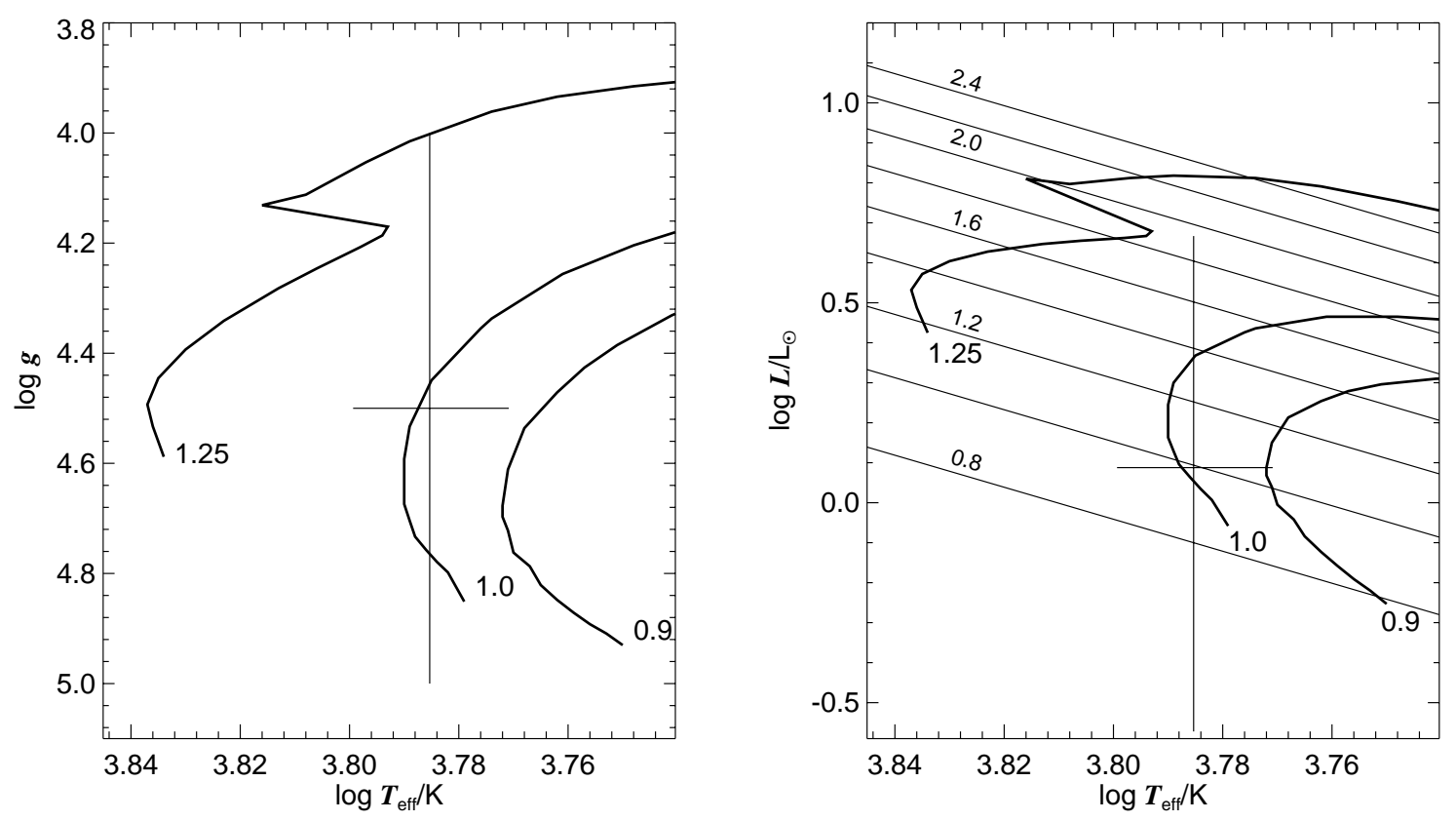

Fig. 5. The parameters of OGLE-TR-3 compared to stellar evolution models (Schaerer et al. 1993). The evolution tracks as well as the lines of constant radii are labeled in solar units.

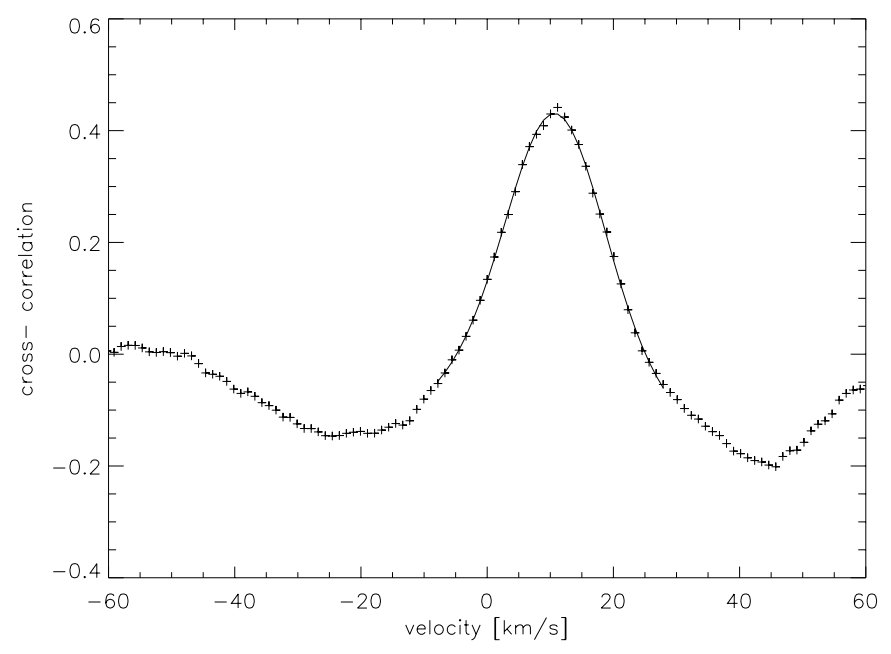

Fig. 6. An example of the cross-correlation. Co-added spectrum versus the one from JD $=2452486.53247$ in the interval [5350 $\AA-5400 \AA]$ $(++++)$ and a Gauss fit (solid line).

derived parameters $\left(R=1.0 R_{\odot}, M=1.0 M_{\odot}\right)$. If we estimate the temperature of the secondary due to irradiation, we derive about $2100 \mathrm{~K}$ for a $6100 \mathrm{~K}$ primary and $5 R_{\odot}$ separation. We utilized the binary eclipse simulation program nightfall (R. Wichmann, Hamburger Sternwarte, Germany) which calculates synthetic light curves taking into account the distortion of the stars in Roche geometry. As can be seen in Fig. 10, we can nicely reproduce the observed $I$-band light curve with an inclination of $90^{\circ}$, a primary radius of $R=1.25 R_{\odot}$ and a secondary radius of $0.14 R_{\odot}(80 \%$ filling factor of the Roche potential of a $0.0006 M_{\odot}$ planet). The radial velocity of the primary is $131 \mathrm{~m} \mathrm{~s}^{-1}$ for such a system, fully consistent with our determined value. The rotational velocity of the primary is $2 \mathrm{~km} \mathrm{~s}^{-1}$ which is far below synchronization $(\approx 5 \%)$. Due to

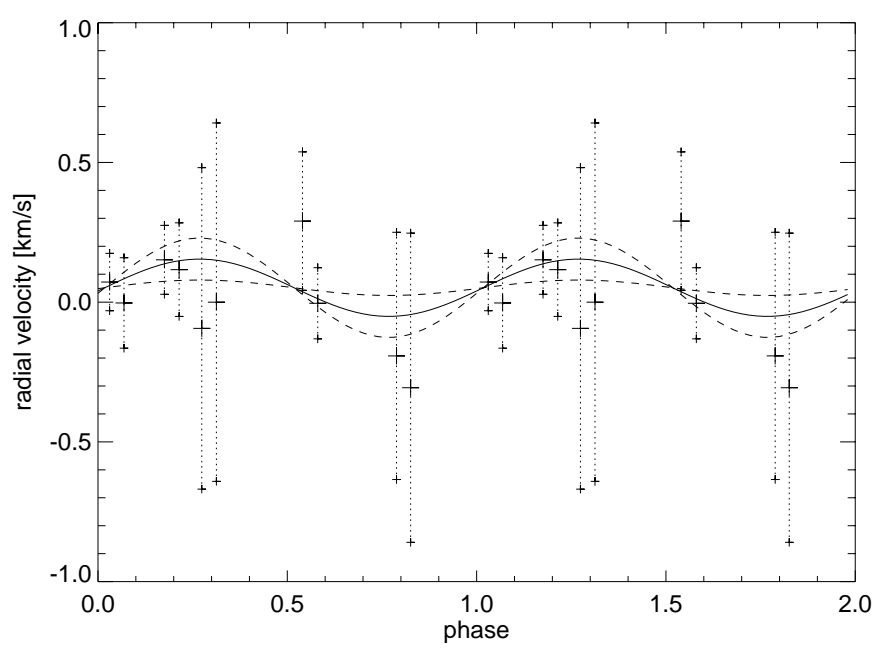

Fig. 7. Measurements of the radial velocity of OGLE-TR-3. Error bars indicate $1 \sigma$ deviations. The solid line is the best fit from our $\chi^{2}$-minimization, the dashed lines are the $3 \sigma$ deviations of the amplitude.

the scatter in the photometry a simultaneous fit of the inclination and the secondary radius is not very well constrained. An inclination of $85^{\circ}$ requires a secondary radius of $0.16 R_{\odot}$, $80^{\circ}$ require $0.17 R_{\odot}$. The formal fit quality, however, favors an inclination very close to $90^{\circ}$. While we derive a radius ratio consistent with that from Udalski et al. (2002a), our absolute radii are slightly smaller than their values $\left(R_{1}=1.48 R_{\odot}, R_{2}=\right.$ $\left.0.18 R_{\odot}\right)$, but within our uncertainty. A very small secondary eclipse might be present in the current photometry. Whether this is real remains to be checked with much more precise photometry. Figure 10 also shows the predicted $H$-band light curve of the secondary eclipse which is of the order of 1 mmag. It should therefore be possible to detect the planet directly with 


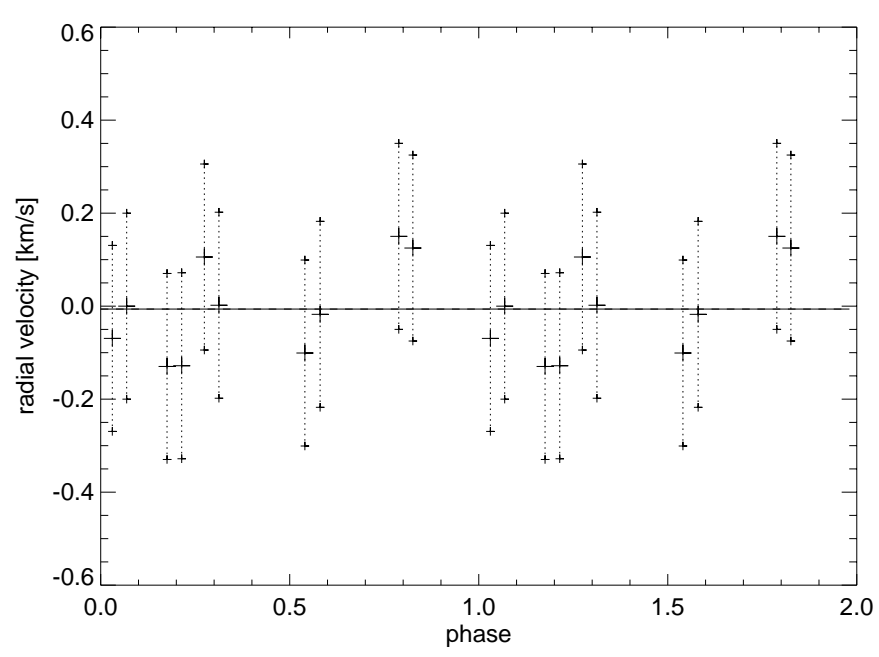

Fig. 8. Measurements of the velocity shift of the night sky emission lines in the spectra of OGLE-TR-3. Error bars indicate $1 \sigma$ deviations. The solid line is the best fit from our $\chi^{2}$-minimization.

Table 2. Results of the radial velocity curve fits. Left block: results from the inversion based $\chi^{2}$-minimization. Right block: results from the genetic algorithm optimization. The blocks give the best fit value and lower and higher limits. Note that the $3 \sigma$ deviation of the pikaia fits are smaller than $1 \%$.

\begin{tabular}{lccc|c}
\hline \hline parameter & \multicolumn{3}{c|}{$\chi^{2}$} & pikaia \\
& best & $-3 \sigma$ & $+3 \sigma$ & best \\
\hline amplitude $\left[\mathrm{km} \mathrm{s}^{-1}\right]$ & 0.10 & 0.08 & 0.13 & 0.12 \\
phase & 0.02 & 0.99 & 0.04 & 0.94 \\
offset $\left[\mathrm{km} \mathrm{s}^{-1}\right]$ & 0.05 & 0.04 & 0.06 & 0.05 \\
\hline
\end{tabular}

high precision IR photometry. We also investigated the effect of the distortion of the primary due to the gravitational field of the secondary. This effect becomes visible in a $\sin (2 P)$-variation outside the eclipses. We fitted both our model solution as well as the observation and find a $0.15 \mathrm{mmag}$ and $0.3 \pm 0.3 \mathrm{mmag}$ variation ( $3 \sigma$ error), respectively. Our solution is therefore consistent with the observations.

While our radial velocity measurements are in favor of the interpretation of OGLE-TR-3 B as planet, the light curve might be explained in the $\mathrm{G}+[\mathrm{M}+\mathrm{M}]$ scenario. The eclipse of the two M2 stars diluted by the G0 star contribution would result in a shallow eclipse of the observed magnitude difference. It requires that the two $\mathrm{M}$ stars are nearly identical. The measured period would correspond to half an orbital phase and the true period of the system would then be $2 \times 1.1899$ days. A period search in the original data, however, results in the largest peak at the period of 1.1899 days. If we simulate the light curve of this system including the third light contribution, we obtain a reasonable fit (Fig. 11), which is, however, not as good as in the case of a transiting planet (Fig. 10). The eclipses are too narrow, the formal fit error is therefore larger than for the planet transit light curve. An increase of the stellar radii to increase the eclipse widths would also influence the depths since the binary would contribute more to the total light. We therefore regard this interpretation for the OGLE-TR-3 system as unlikely.
The $\mathrm{G}+[\mathrm{M}+\mathrm{M}]$ scenario can easily be checked, since the $\mathrm{M}$ star contribution to the total light is strongly wavelength dependent. Hence, we would expect a strong color effect in the light curve.

The $\mathrm{G}+[X+Y]$ blending scenario would also require a false detection of the radial-velocity variation. Furthermore, we checked possible blends for their consistency with the light curve. Assuming that the secondary is much fainter than the primary (no detectable secondary eclipse), we can pick a spectral type of the secondary with the corresponding radius and mass for a main sequence star. Unblended light curves for various contributions from a less distant G0 star are reconstructed, the depth of the primary eclipse then provides the radius of the primary. The corresponding mass together with the period is used to derive the orbital separation and velocity (Kepler's third law, assuming circular orbits). The time between first and fourth contact can then be obtained from the orbital velocity and the sum of the radii, the time between second and third contact from the orbital velocity and the difference of the radii. In Fig. 12 these eclipse times are plotted in units of the corresponding times obtained from the observed light curve. A consistent solution is only possible in a very limited parameter range: The foreground G0 star provides about $80 \%$ of the total light, the eclipsing binary system is then a F9 secondary and a B9 primary. However, this scenario can be ruled out when a theoretical light curve is calculated with these parameters. The close vicinity of the two stars would result in an observable sinusoidal variation. Additionally, the light curve can only be reproduced very roughly with this solution. We therefore reject the $\mathrm{G}+[X+Y]$ scenario based on our current observational data.

\section{Summary and discussion}

Summarizing, our data favor the interpretation of OGLE-TR-3 as planetary host star and we have detected the radial velocity variation due to the reflex motion of the unseen planetary companion.

From Kepler's third law together with momentum conservation the companion mass can be determined from the period (1.1899 days, Udalski et al. 2002a), the mass of the primary $\left(1.0 M_{\odot}\right.$, this paper), the velocity amplitude $\left(120 \mathrm{~m} \mathrm{~s}^{-1}\right.$, this paper), and the inclination, which must be close to $90^{\circ}$ (see above, in the following we will use $90^{\circ}$ ).

The upper limit of the derived radius $\left(R=0.17 R_{\odot}\right)$ of the unseen companion would be consistent with an M star down to a gas-giant planet. With our VLT observations, however, we can clearly rule out an $\mathrm{M}$ star since a $0.08 M_{\odot}$ star would cause a $15 \mathrm{~km} \mathrm{~s}^{-1}$ reflex velocity variation. We can also rule out a brown dwarf companion since a brown dwarf at the lower limit for deuterium burning $\left(0.013 M_{\odot}\right)$ would still result in $2.5 \mathrm{~km} \mathrm{~s}^{-1}$ velocity amplitude. As a conservative upper limit for velocity variations $(3 \sigma)$ we derive $500 \mathrm{~m} \mathrm{~s}^{-1}$ which translates into $0.0026 M_{\odot}$. If our fit is regarded as reliable, we obtain a mass of 0.0005 to $0.0006 M_{\odot}$ or about half a Jupiter mass. Due to the small period, the separation of the two objects is only about $5 R_{\odot}$ which is however still a factor of two above the Roche stability limit. The companion's mean mass density is comparable to the one of HD 209458 B. If we use 

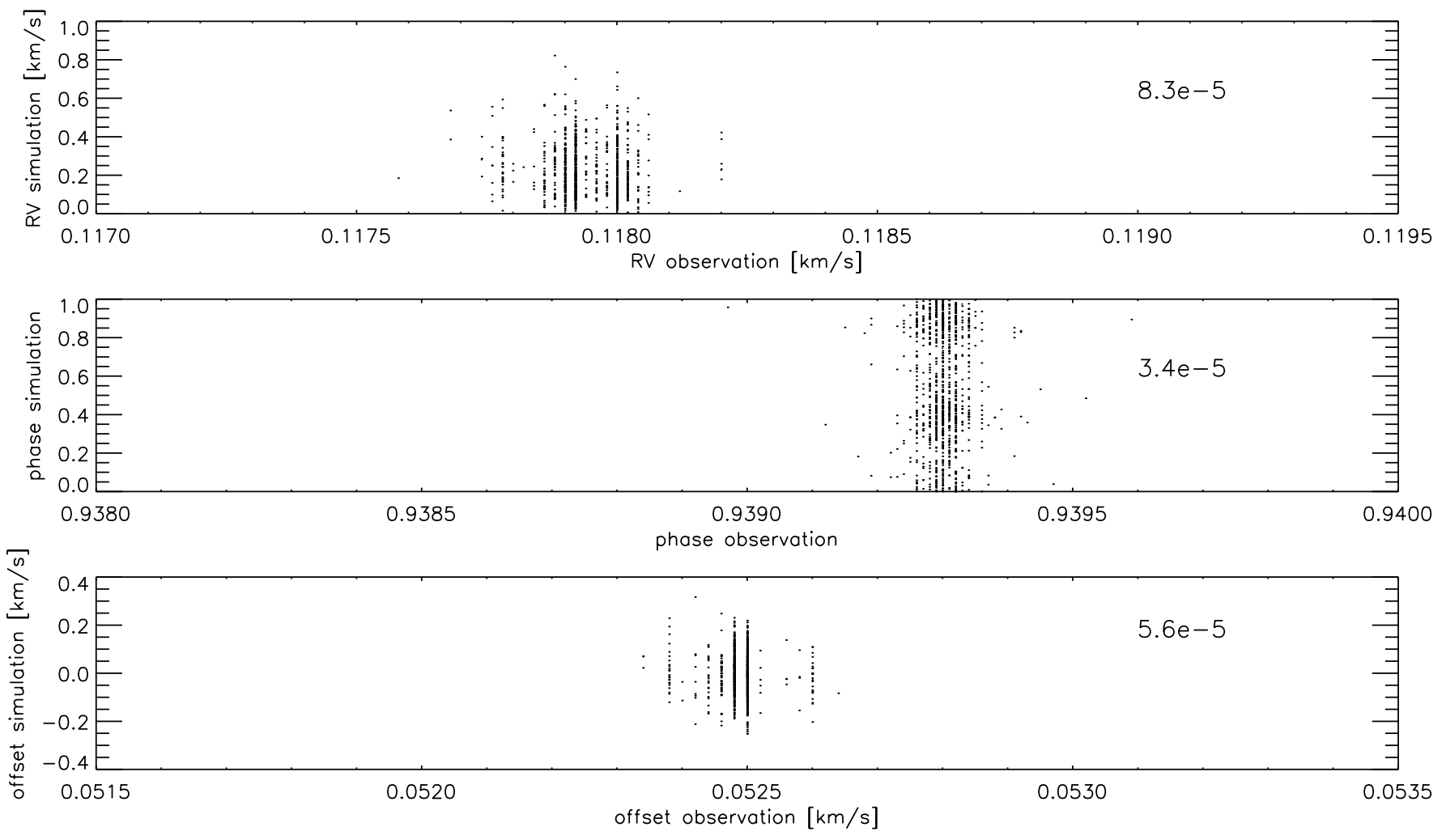

Fig. 9. Fit results of synthetic white noise curves versus fit results of the OGLE-TR-3 radial velocity $(R V)$ curve using pikaia. While the synthetic data (ordinate) spread over a wide interval, the real data (abscissa) are confined to a narrow range only. The ratio between the distribution widths of the fits is indicated in the plots.
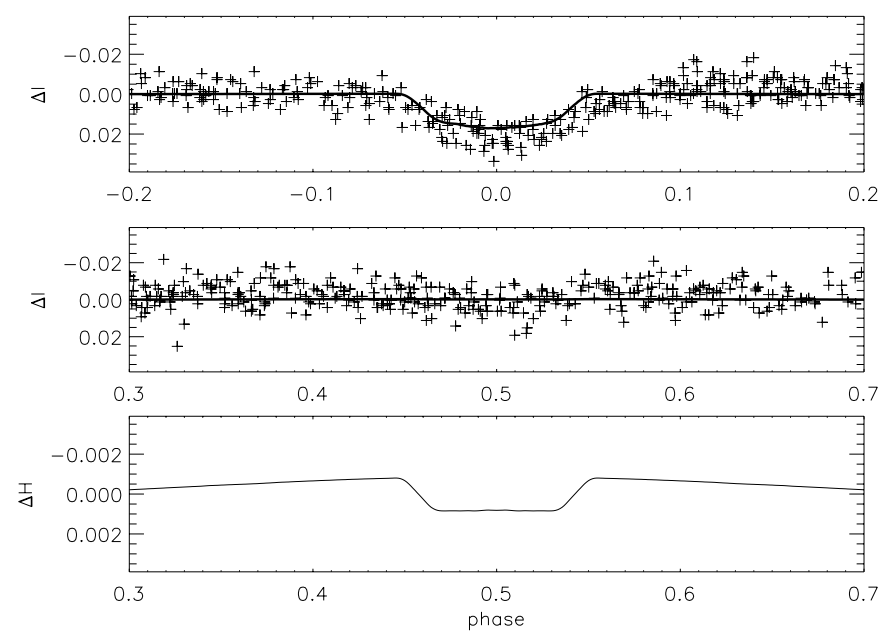

Fig. 10. Light curve of OGLE-TR-3 from Udalski et al. (2002a): top and middle: $I$ band observed $(++++)$ and synthetic light curve (solid line). Bottom: predicted $H$ band light curve at a smaller scale. The primary eclipse is shown in the top panel, the secondary eclipse in the lower two.

our $0.0005 M_{\odot}$ and $0.014 R_{\odot}$, it amounts to $0.25 \mathrm{~g} \mathrm{~cm}^{-3}$, which is about $20 \%$ of the mean solar density.

The shortest known orbital period of all extrasolar planets and the very close orbit of OGLE-TR-3 raises the question of the influence of tidal effects between the star and planet. As the star appears to be rotating much slower than the planetary orbital period, any tides raised by the planet on the star will lead
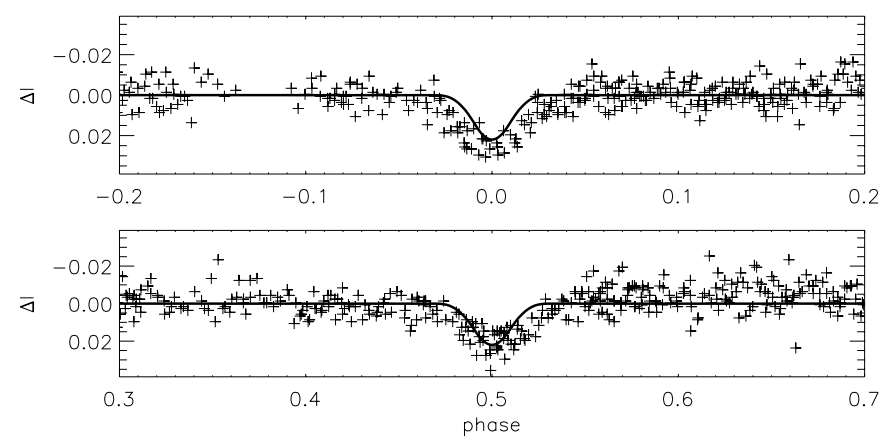

Fig. 11. Light curve of OGLE-TR-3 from Udalski et al. (2002a): $I$ band observed $(++++)$ and synthetic light curve (solid line). The photometric data are folded with $2 \times 1.1899$ days in order to simulate the $\mathrm{G}+[\mathrm{M}+\mathrm{M}]$ scenario. The eclipsing system (primary minimum top, secondary minimum bottom panel) consists of two M2 stars; the third light contribution is that from a $\mathrm{G}$ star.

to an additional shrinking of the orbit. Following the argmuments of Goldreich \& Soter (1966) we estimate the timescale $a / \dot{a}$ for a change in the semi-major axis of the planet, using the present orbital parameter. Using a value of $10^{7}$ for the dissipation factor $Q_{*}$ of the star we obtain a damping timescale of about $2 \times 10^{9}$ yrs. As the $Q$ value for stars is quite uncertain and may easily be higher, we may conclude that a planet is just about able to survive in such a close orbit for the lifetime of the star. 


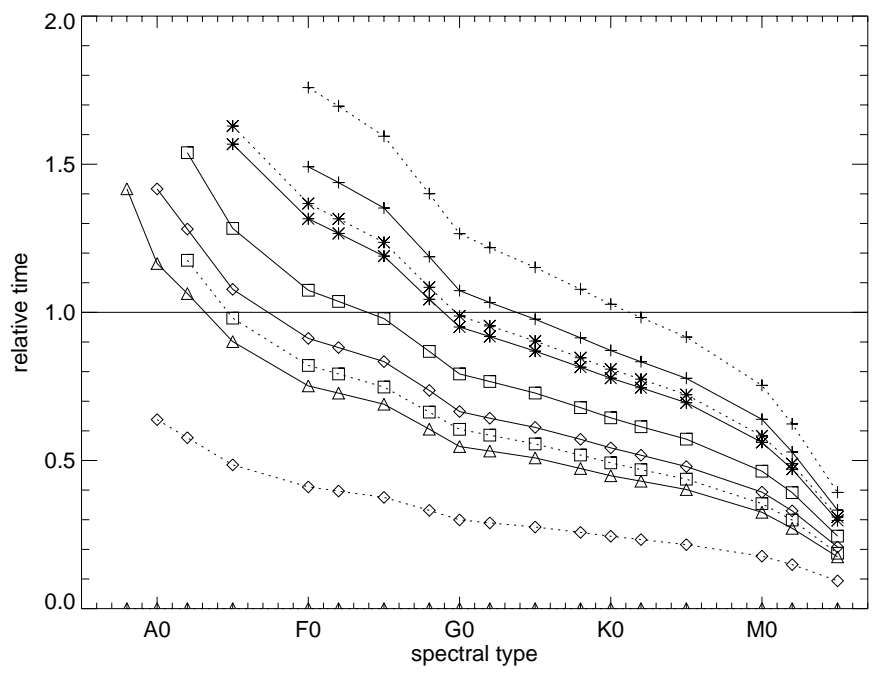

Fig. 12. Predicted eclipse durations for blending scenarios as function of the spectral type of the secondary (first to fourth contact: full line; second to third contact dashed line) in units of eclipse durations of OGLE-TR-3. Contribution from a foreground star with 70, 80, 90, 95, $98 \%$ is labeled with crosses, stars, squares, diamonds, and triangles respectively.

We therefore conclude that we confirmed the lowluminosity companion of OGLE-TR-3 as an extrasolar planet $\left(M_{1}=1.0 M_{\odot}, M_{2}=0.0005 M_{\odot}\right.$ and $\left.R_{2}=0.14 R_{\odot}\right)$ with quite similar properties compared to HD $209458\left(M_{1}=1.05 M_{\odot}\right.$, $\left.M_{2}=0.00066 M_{\odot}, R_{2}=0.147 R_{\odot}\right)$ and OGLE-TR-56 $\left(M_{1}=\right.$ $\left.1.04 M_{\odot}, M_{2}=0.00086 M_{\odot}, R_{2}=0.13 R_{\odot}\right)$ regarding the parameters of the primary star and planetary companion. The system of OGLE-TR-3 is slightly more extreme regarding the separation.

Finally, if confirmed, our results for OGLE-TR-3, together with those for OGLE-TR-56, make these objects the first two extrasolar planets detected via the transiting method. A further confirmation of the planetary nature would benefit from several supporting observations: optical $B, V, R$ photometry to exclude a color effect expected in the blending scenarios, IR spectroscopy to exclude an M star contribution, $H$ band photometry to detect the predicted very shallow secondary eclipse, and finally, more radial velocity measurements to detect the orbital period in the radial velocity data directly.

Acknowledgements. We want to express our thanks to the OGLE team lead by A. Udalski, who provided their photometry which is the basis of all the follow-up spectroscopy presented here. We like to thank the referee and A. Hatzes for very useful comments. The UVES spectra used in this analysis were obtained as part of an ESO Service Mode run, proposal 269.C-5034. We acknowledge the use of the nightfall program for the light-curve synthesis of eclipsing binaries (http://www.lsw. uni-heidelberg.de/ rwichman/ Nightfall.html), written by Rainer Wichmann. This research was supported in part by the DLR under grant 50 OR 0201 (Tübingen) and in part by NSF grants AST-9720704 and AST-0086246, NASA grants NAG5-8425, NAG5-9222, as well as NASA/JPL grant 961582 to the University of Georgia. This work was supported in part by the Pôle Scientifique de Modélisation Numérique at ENS-Lyon. Some of the calculations presented in this paper were performed on the IBM pSeries 690 of the Norddeutscher Verbund für Hochund Höchstleistungsrechnen (HLRN), on the IBM SP "seaborg" of the NERSC, with support from the DoE, and on the IBM SP "Blue Horizon" of the San Diego Supercomputer Center (SDSC), with support from the National Science Foundation. We thank all these institutions for a generous allocation of computer time.

\section{References}

Allard, F., Hauschildt, P. H., Alexander, D. R., Tamanai, A., \& Schweitzer, A. 2001, ApJ, 556, 357

Charbonneau, P. 1995, ApJS, 101, 309

Charbonneau, D., Brown, T. M., Latham, D., \& Mayor, M. 2000, ApJ, 529, L45

Charbonneau, D., Brown, T. M., Noyes, R. W., \& Gilliland, R. L. 2002, ApJ, 568, 377

Dreizler, S., Rauch, T., Hauschildt, P., et al. 2002, A\&A, 391, L17

Goldreich, P., \& Soter, S. 1966, Icarus, 5, 375

Hauschildt, P. H., Allard, F., Ferguson, J., Baron, E., \& Alexander, D. R. 1999, ApJ, 525, 871

Henry, G. W., Marcy, G. W., Butler, R. P., \& Vogt, S. S. 2000, ApJ, 529, L41

Konacki, M., Torres, G., Jha, S., \& Sasselov, D. D. 2003, Nature, 421, 507

Mayor, M., \& Queloz, D. 1995, Nature, 378, 355

Mazeh, T., Naef, D., Torres, G., et al. 2000, ApJ, 532, L55

Schaerer, D., Meynet, G., Maeder, A., \& Schaller, G. 1993, A\&AS, 98,523

Schneider, J. 2001, Encyclopedia of Astron. \& Astrophys., ed. P. Murdin (London: IoP Publishing), 759

Udalski, A., Paczynski, B., Zebrun, K., et al. 2002a, Acta Astron., 52,1

Udalski, A., Zebrun, K., Szymanski, M., et al. 2002b, Acta Astron., 52,115

Wolszczan, A., \& Frail, D. A. 1992, Nature, 355, 145 